\title{
Extensive Acute Lower Extremity Arterial Thrombosis: A Major Thrombus Formation Caused by COVID-19
}

\author{
Pouya Tayebi ${ }^{1}$, Mahmoud Sadeghi Haddad Zavareh ${ }^{2}$, Gooya Tayyebi ${ }^{3}$, Fatemeh Zahra Abdollahi ${ }^{4}$, \\ and Fatemeh Mahmoudlou ${ }^{4}$ \\ 'Department of Vascular and Endovascular Surgery, Rouhani Hospital, ${ }^{2}$ Infectious Diseases and Tropical Medicine Research Center, \\ Health Research Institute, Babol University of Medical Sciences, Babol, ${ }^{3}$ Department of Geriatric Psychiatry, Rasoul Akram Hospital, \\ Iran University of Medical Sciences, Tehran, ${ }^{4}$ Student Research Committee, Babol University of Medical Sciences, Babol, Iran
}

\begin{abstract}
Acute thromboembolic events have been frequently reported in patients with coronavirus disease 2019 (COVID-19) due to an increase in the coagulation system activity and endothelial dysfunction. This report describes a patient with COVID-19 who initially reported respiratory symptoms and developed acute lower limb ischemia secondary to extensive macrovascular arterial thrombosis, which was treated with thrombectomy. The development of such extensive arterial thrombosis with anticoagulants at therapeutic doses is a new sign of increased viral pathogenicity, and it is necessary to develop and apply updated prophylaxis protocols for thrombosis in these patients.
\end{abstract}

Key Words: Acute limb ischemia, Arterial thrombosis, COVID-19
Received May 31, 2021

Revised August 3, 2021

Accepted September 9, 2021

Published on November 22, 2021

Corresponding author: Mahmoud Sadeghi Haddad Zavareh

Infectious Diseases and Tropical Medicine Research Center, Babol University of Medical Sciences, Keshavarz Boulevard, Babol 4717641367, Iran

Tel: 989381915009

Fax: 981132238300

drm_sadeghihz@yahoo.com

https://orcid.org/0000-0001-8701-7267

Copyright $\odot 2021$ The Korean Society for Vascular Surgery

This is an Open Access article distributed under the terms of the Creative Commons Attribution Non-Commercial License (http://creativecommons.org/licenses/by-nc/4.0) which permits unrestricted non-commercial use, distribution, and reproduction in any medium, provided the original work is properly cited.

Cite this article; Vasc Specialist Int 2021. https://doi.org/10.5758/vsi.210039

\section{INTRODUCTION}

After the outbreak of coronavirus disease 2019 (COVID-19) worldwide, various clinical manifestations have been reported in infected patients. In addition to the common respiratory manifestations of the disease, thromboembolic events have also been described [1]. Studies have shown that COVID-19 can increase the levels and activities of proand anti-inflammatory cytokines [2,3]. There is also evidence of increased complement activity in these patients, secondary to the viral infection of vascular endothelial cells and increased secretion of anaphylatoxin C5a [4]. Such biological changes in the patient's body, which lead to increased blood coagulation, have already been confirmed by the presence of microclots in the lung microvessels during the lung autopsies of COVID-19 patients [5]. However, the microclots can turn into larger thrombi, which can lead to thromboembolic events, such as stroke, deep vein thrombosis, pulmonary embolism, and arterial ischemia [6]. The severity of thrombosis in the main arteries of the limbs is usually short [7], but in our patient, a very large thrombosis occurred in the arteries of the lower limbs, which was unique in its kind.

Informed consent was obtained from the patient's family for publication, and this was approved by the ethics committee with reference (no. IR.MUBABOL.REC.1400.100). 


\section{CASE}

An 80-year-old male patient with a history of diabetes mellitus presented to the emergency department complaining of shortness of breath and cough. His vital signs on presentation were as follows: temperature, $37^{\circ} \mathrm{C}$; heart rate, 112 beats/min; blood pressure, 120/75 mmHg; respiratory rate, 28 breaths/min; and oxygen saturation, 90\% on supplemental oxygen. There were no significant points in the general examination. His bilateral lower extremities were normal, and his bilateral popliteal, posterior tibial, and dorsalis pedis arterial pulses were palpable. On laboratory testing, the following values were noted: white blood cells, $7 \times 10^{9} / \mathrm{L}$ (reference, $4.5 \times 10^{9}-11.5 \times 10^{9}$ ); hemoglobin, $13.6 \mathrm{~g} / \mathrm{dL}$ (12-16); hematocrit, 37.3\% (35\%-45\%); platelets, $210 \times 10^{9} / \mathrm{L}\left(140 \times 10^{9}-440 \times 10^{9}\right)$; glucose, $378 \mathrm{mg} / \mathrm{dL}(70-$ 105); creatinine, $1.4 \mathrm{mg} / \mathrm{dL}$ (0.6-1.40); blood urea nitrogen, $11.3 \mathrm{mg} / \mathrm{dL}$ (7-23); D-dimer, $248 \mathrm{ng} / \mathrm{mL}$ (<0.5); prothrombin time, $20 \mathrm{sec}$ (12.2-14.9); international normalized ratio, 1.1 (<1); partial thromboplastin time, $40 \mathrm{sec}(21.3-35.1)$; Creactive protein, $151 \mathrm{mg} / \mathrm{L}(<10 \mathrm{mg} / \mathrm{L})$; and erythrocyte sedimentation rate, $88 \mathrm{~mm} / \mathrm{h}(0-32)$; lupus anticoagulant, negative; interleukine-6, $76 \mathrm{pg} / \mathrm{mL}$ (5-15). His nasopharyngeal swab was positive for the severe acute respiratory syndrome coronavirus-2, and high-resolution computed tomography showed bilateral extensive interstitial lung infiltrates. As such, the patient was admitted to the intensive care unit (ICU) and was treated with Ziferon (Interferon-1b, 250 mg/mL; Zist Daru Danesh, Tehran, Iran) every two days via subcutaneous administration, dexamethasone $8 \mathrm{mg} /$ day

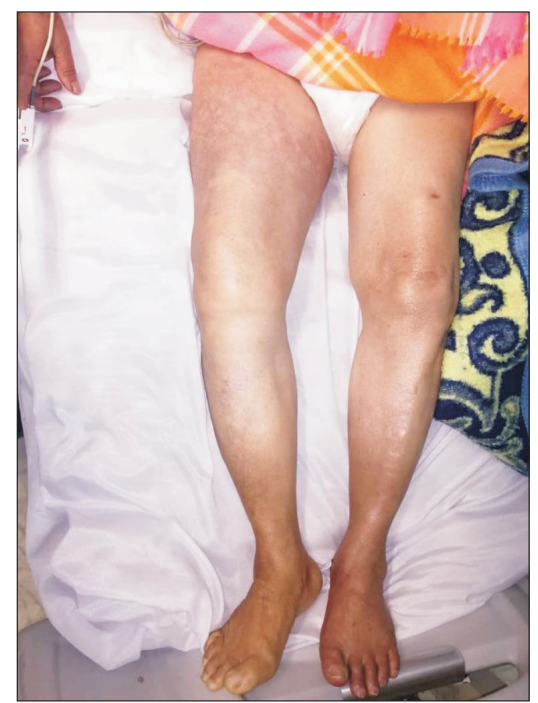

Fig. 1. The figure showed the discoloration of the right lower limb due to acute limb ischemia in a severely ill COVID-19 patient. via intravenous administration, Remdesivir $(100 \mathrm{mg} / 20 \mathrm{~mL}$; Actoverco, Tehran, Iran) $200 \mathrm{mg}$ on the first day followed by $100 \mathrm{mg} /$ day, and heparin 5,000 units subcutaneously every 6 hours. On the ninth day after admission, with the patient still complaining of shortness of breath $\mathrm{O}_{2}$ saturation: 89\%), he experienced sudden pain in the right lower extremity with coldness, ischemic discoloration, paralysis, and lack of femoral pulses of the limb (Fig. 1). Computed tomography angiography revealed the total occlusion of the right common iliac artery with no distal run-off (Fig. 2). Electrocardiography did not show any arrhythmia, and the platelet count was normal on the day of the event and after. The patient underwent an emergency thromboembolectomy with spinal anesthesia via a femoral incision. A long thrombus with a cohesive, tubular consistency emerged from the iliac, femoral, and tibial arteries (Fig. 3, Supplementary Video 1). After the successful thrombectomy, the ischemia resolved, and the patient was transferred to the ICU. In the ICU, the patient experienced respiratory acidosis $\left(\mathrm{pH}, 7.27 ; \mathrm{PCO}_{2}, 55 ; \mathrm{PO}_{2}, 90 ; \mathrm{HCO}_{3}, 14.4\right.$; base excess, -2.1) but had adequate urine flow. He also received intravenous heparin at a dose of 1,000 units per hour. The right lower extremity was warm, and the symptoms of ischemia resolved. In addition, necessary measures were taken to prevent reperfusion syndrome. Ten hours after the operation, the patient's respiratory distress worsened despite receiving $100 \%$ oxygen. He eventually suffered from cardiopulmonary arrest and died.

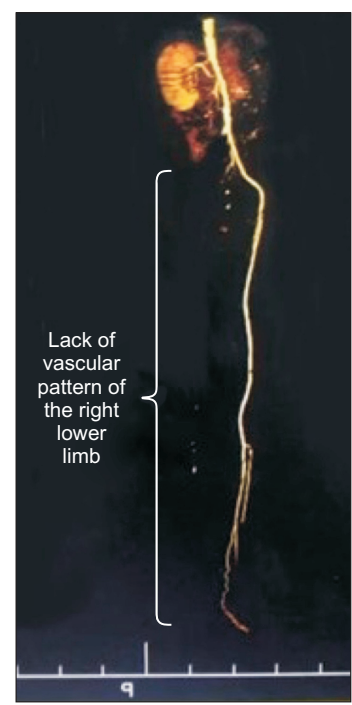

Fig. 2. The reconstructed computed tomography angiography revealed a complete arterial thrombosis in the right lower extremity. 


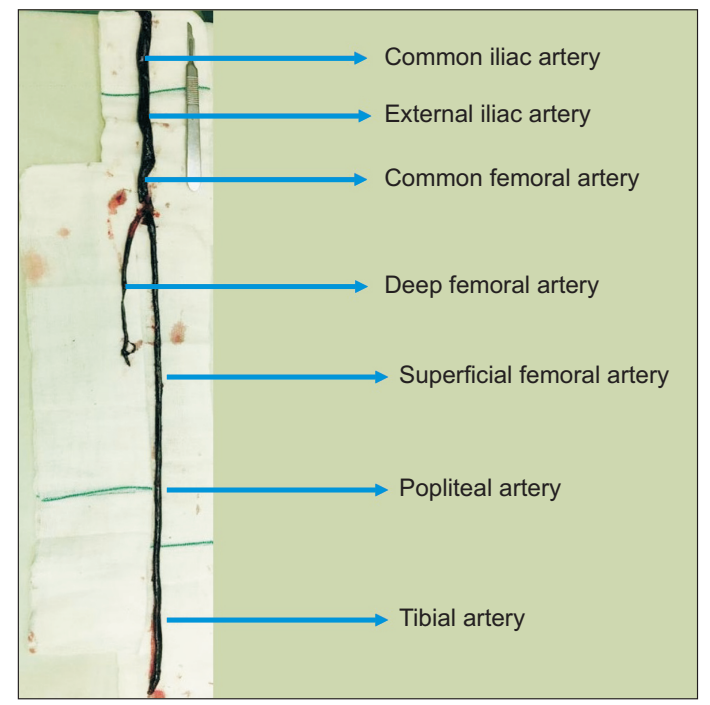

Fig. 3. Surgically-removed long clots are displayed according to the artery of origin.

\section{DISCUSSION}

Venous thrombosis and pulmonary embolism have been reported frequently in patients with COVID-19, but acute limb ischemia secondary to acute arterial thrombosis has been less commonly described. Studies have shown that hypercoagulability and endothelial damage in these patients are the main causes of thromboembolic events [8]. The presence of persistent lupus anticoagulation and high D-dimer levels in patients with COVID-19 increases the risk of associated thrombosis $[9,10]$. The remarkable incident about this patient was that he had an acute arterial thromboembolic accident despite being on therapeutic anticoagulation. Although the exact cause of this accident is unknown, the large thrombus (from the proximal common iliac artery to the distal leg arteries) may have been formed due to the increased coagulation status or endothelial damage secondary to COVID-19. Despite the normal results of coagulation tests or the administration of therapeutic anticoagulants, thrombotic events are likely in COVID-19 patients. Furthermore, during inflammatory storms, an increase in inflammatory mediators has been reported, which cannot be assessed by routine coagulation tests [6]. Immobility in critically ill COVID-19 patients can also lead to thrombotic events. The combination of these conditions with an increase in acute-phase reactants such as interleukin-6, which can cause endothelial damage and endothelial dysfunction, may justify the extensive thrombosis in the patient's arterial system [11]. However, it is interesting to note how the thrombosis occurred in the arterial system, where there is a high rate of blood flow, when it would have been more logical for it to occur in the venous system, where the chance of blood stasis is higher. Anticoagulants are now an important treatment option for patients with severe COVID-19. The results of a study by Tang et al. [12] showed that the administration of heparin at a therapeutic dose reduced mortality by approximately $20 \%$ in patients with D-dimer levels greater than $3000 \mathrm{ng} / \mathrm{mL}$ over 28 days. Although starting anticoagulants has been shown to be beneficial in cases where the D-dimer level is six times higher than normal, it is unclear whether it will still provide additional benefits if higher doses are used [12]. In conclusion, it is not yet clear how thrombosis develops in critically ill patients with COVID-19. The occurrence of these complications while receiving anticoagulants in these patients indicates that the administration of anticoagulants cannot prevent acute arterial thrombosis. Therefore, the authors suggest that further research on the pathogenesis and management of COVID-19-induced hypercoagulable states should be conducted.

\section{ACKNOWLEDGEMENTS}

We thank the nurses at the vascular and endovascular surgery and the infectious disease unit of the Ayatollah Rouhani Hospital of Babol University of Medical Sciences.

\section{FUNDING}

None.

\section{CONFLICTS OF INTEREST}

The authors have nothing to disclose.

\section{ORCID}

Pouya Tayebi
https://orcid.org/0000-0002-6923-0221
Mahmoud Sadeghi Haddad Zavareh
https://orcid.org/0000-0001-8701-7267
Gooya Tayyebi
https://orcid.org/0000-0001-5516-0798
Fatemeh Zahra Abdollahi
https://orcid.org/0000-0003-3885-3159
Fatemeh Mahmoudlou
https://orcid.org/0000-0002-8251-9329

\section{AUTHOR CONTRIBUTIONS}

Concept and design: PT, MSHZ. Analysis and interpretation: not applicable. Data collection: PT, MSHZ, GT. Writing 
the article: PT, MSHZ, FZA, FM, GT. Critical revision of the article: PT, MSHZ, FZA, FM. Final approval of the article:
PT. Statistical analysis: not applicable. Obtained funding: not applicable. Overall responsibility: MSHZ, PT.
1) Mondal S, Quintili AL, Karamchandani K, Bose S. Thromboembolic disease in COVID-19 patients: a brief narrative review. J Intensive Care 2020;8:70.

2) Faix JD. Biomarkers of sepsis. Crit Rev Clin Lab Sci 2013;50:23-36.

3) Yang Y, Shen C, Li J, Yuan J, Wei J, Huang F, et al. Plasma IP-10 and MCP3 levels are highly associated with disease severity and predict the progression of COVID-19. J Allergy Clin Immunol 2020;146:119-127.e4.

4) Gao T, Hu M, Zhang X, Li H, Zhu $\mathrm{L}$, Liu $\mathrm{H}$, et al. Highly pathogenic coronavirus $\mathrm{N}$ protein aggravates lung injury by MASP-2-mediated complement over-activation. MedRxiv 2020.03.29.20041962 [Preprint]. 2020 [cited 2020 Jan 1]. Available from: https://doi.org/10.1101/2020.03.29.200 41962.

5) Ciceri F, Beretta L, Scandroglio AM, Colombo S, Landoni G, Ruggeri A, et al. Microvascular COVID-19 lung vessels obstructive thromboinflammatory syndrome (MicroCLOTS): an atypical acute respiratory distress syndrome working hypothesis. Crit Care Resusc 2020;22:95-97.

6) Magro C, Mulvey JJ, Berlin D, Nuovo G, Salvatore S, Harp J, et al. Complement associated microvascular injury and thrombosis in the pathogenesis of severe COVID-19 infection: a report of five cases. Transl Res 2020;220:1-13.

7) Klok FA, Kruip MJHA, van der Meer NJM, Arbous MS, Gommers DAMPJ, et al. Incidence of thrombotic complications in critically ill ICU patients with COVID-19. Thromb Res 2020;191:145147.

8) Bellosta R, Luzzani L, Natalini G, Pegorer MA, Attisani L, Cossu LG, et al. Acute limb ischemia in patients with COVID-19 pneumonia. J Vasc Surg 2020;72:1864-1872.
9) Bowles L, Platton S, Yartey N, Dave M, Lee K, Hart DP, et al. Lupus anticoagulant and abnormal coagulation tests in patients with COVID-19. N Engl J Med 2020;383:288-290.

10) Cui S, Chen S, Li X, Liu S, Wang F. Prevalence of venous thromboembolism in patients with severe novel coronavirus pneumonia. J Thromb Haemost 2020;18:1421-1424.

11) Halici B, Sarinc Ulasli S, Günay E, Nural S, Sen S, Akar 0, et al. Assessment of inflammatory biomarkers and oxidative stress in pulmonary thromboembolism: follow-up results. Inflammation 2014;37:1186-1190.

12) Tang N, Bai H, Chen X, Gong J, Li D, Sun Z. Anticoagulant treatment is associated with decreased mortality in severe coronavirus disease 2019 patients with coagulopathy. J Thromb Haemost 2020;18:1094-1099. 